\title{
Conhecimento dos enfermeiros acerca da importância do uso de protocolos de cuidados: Discurso do sujeito coletivo
}

Knowledge of nurses about the importance of the use of care protocols: Collective subject discourse Conhecimento dos enfermeiros acerca da importância do uso de protocolos de cuidados: Discurso do sujeito coletivo

Recebido: 14/12/2020 | Revisado: 22/12/2020 | Aceito: 04/01/2021 | Publicado: 06/01/2021

Rayra Mass Lucena de Sena Lima

ORCID: https://orcid.org/0000-0002-9408-6401 Universidade Federal do Rio Grande do Norte, Brasil E-mail: rayra.mass@hotmail.com

Micheline Veras de Moura

ORCID: https://orcid.org/0000-0001-8519-637X

Universidade de Coimbra, Portugal Universidade de Brasília, Brasil

E-mail: michelinehenriques@yahoo.com.br

Johnata da Cruz Matos

ORCID: https://orcid.org/0000-0002-3359-4437 Universidade de Brasília, Brasil

E-mail: johnata.matos@hotmail.com

Karla Cristina Walter

ORCID: https://orcid.org/0000-0002-5622-0198 Universidade de Coimbra, Portugal E-mail: karla.cris@imepac.edu.br

Aliete Cristina Gomes Dias Pedrosa da Cunha Oliveira

ORCID: https://orcid.org/0000-0001-8399-8619 Universidade de Coimbra, Portugal E-mail: aliete.cunha3@gmail.com

Verbena Santos Araújo

ORCID: https://orcid.org/0000-0002-3519-4744 Universidade Federal do Rio Grande do Norte, Brasil E-mail: verbena@ufrn.edu.br

Eliane Santos Cavalcante

ORCID: https://orcid.org/0000-0002-0001-9161 Universidade Federal do Rio Grande do Norte, Brasil E-mail: elianeufrn@hotmail.com

Vilani Medeiros de Araújo Nunes ORCID: https://orcid.org/0000-0002-9547-0093 Universidade Federal do Rio Grande do Norte, Brasil E-mail: vilani@ufrn.edu.br

Michel Siqueira da Silva

ORCID: https://orcid.org/0000-0002-0391-3249 Centro Universitário FACEX, Brasil

E-mail: michelsiqueira10@gmail.com

Maria Socorro Morais Sisnando

ORCID: https://orcid.org/0000-0001-5139-3682

Maternidade Escola Assis Chateaubriand, Brasil

E-mail: socorrosisnando@hotmail.com

\begin{abstract}
Resumo
Objetivo: averiguar o conhecimento de enfermeiros intensivistas acerca da prevenção e avaliação de risco relacionados a LPP e a importância da utilização de protocolos de cuidados de enfermagem. Métodos: Trata-se de estudo exploratório-descritivo com abordagem qualitativa. Os participantes do presente estudo são compostos por enfermeiros intensivistas assistenciais, enfermeira coordenadora da UTI e gerente de enfermagem, além das enfermeiras responsáveis pela Comissão de Curativos e Segurança do Paciente, totalizando dez participantes. Os dados foram coletados entre março e dezembro de 2019. Foram aplicadas entrevistas semiestruturadas individuais e posteriormente, as respostas dos entrevistados foram transcritas na íntegra e analisadas através do Discurso do Sujeito Coletivo. Resultados: A análise dos depoimentos foi baseada em seis questionamentos, que resultaram em nove DSC. As respostas foram subdividas em "Categoria A" e "Categoria B" quando dois tipos de respostas diferentes foram obtidos. Com isso, os questionamentos 1, 2 e 3 apresentam DSC nas categorias A e B, enquanto os questionamentos 4, 5 e 6 apenas apresentam DSC na "Categoria A". Conclusão: O estudo possibilitou uma melhor compreensão das
\end{abstract}


condições vivenciadas pelos enfermeiros que atuam em UTI apontando potencialidades, dificuldades e limitações na prevenção e tratamento as LPP.

Palavras-chave: Lesão por pressão; Unidade de terapia intensiva; Segurança do paciente.

\begin{abstract}
Objective: to investigate the knowledge of intensive care nurses about prevention and risk assessment related to PPL and the importance of using nursing care protocols. Methods: This is an exploratory-descriptive study with a qualitative approach. The participants in this study are composed of intensive care nurses, ICU coordinating nurse and nursing manager, in addition to nurses responsible for the Dressing and Patient Safety Commission, totaling ten participants. Data were collected between March and December 2019. Individual semi-structured interviews were applied and later, the respondents' responses were transcribed in full and analyzed through the Collective Subject Discourse. Results: The analysis of the statements was based on six questions, which resulted in nine CSDs. The responses were subdivided into "Category A" and "Category B" when two different types of responses were obtained. As a result, questions 1, 2 and 3 show CSD in categories A and B, while questions 4, 5 and 6 only have CSD in "Category A". Conclusion: The study enabled a better understanding of the conditions experienced by nurses working in the ICU, pointing out potentials, difficulties and limitations in the prevention and treatment of PPL.
\end{abstract}

Keywords: Pressure ulcer; Intensive care units; Patient safety.

\title{
Resumen
}

Objetivo: investigar el conocimiento de los enfermeros de cuidados intensivos sobre la prevención y evaluación de riesgos relacionados con la LPP y la importancia del uso de protocolos de atención de enfermería. Métodos: Se trata de un estudio exploratorio-descriptivo con enfoque cualitativo. Los participantes de este estudio están compuestos por enfermeras de cuidados intensivos, enfermera coordinadora de UCI y gerente de enfermería, además de enfermeras responsables de la Comisión de Vestuario y Seguridad del Paciente, totalizando diez participantes. Los datos fueron recolectados entre marzo y diciembre de 2019. Se aplicaron entrevistas semiestructuradas individuales y posteriormente, las respuestas de los encuestados fueron transcritas íntegramente y analizadas a través del Discurso Colectivo del Sujeto. Resultados: El análisis de los enunciados se basó en seis preguntas, que resultaron en nueve DCS. Las respuestas se subdividieron en "Categoría A" y "Categoría B" cuando se obtuvieron dos tipos diferentes de respuestas. Como resultado, las preguntas 1, 2 y 3 muestran CSD en las categorías A y B, mientras que las preguntas 4, 5 y 6 solo tienen CSD en la "Categoría A". Conclusión: El estudio permitió conocer mejor las condiciones que viven las enfermeras que laboran en la UCI, señalando potenciales, dificultades y limitaciones en la prevención y tratamiento de la LPP.

Palabras clave: Úlcera por presión; Unidades de cuidados intensivos; Seguridad del paciente.

\section{Introdução}

A Segurança do Paciente (SP) foi definida pela Portaria $n^{\circ} 529$, de $1^{\circ}$ de abril de 2013 como uma redução, a um mínimo aceitável, do risco de dano desnecessário associado ao cuidado de saúde (Brasil, 2013).

As primeiras publicações sobre o assunto ocorreram no ano de 1999 pelo Instituto de Medicina (IOM) dos Estados Unidos da América (EUA), onde foi detectado que entre 44 mil e 98 mil pacientes morriam a cada ano nos hospitais dos EUA em virtude de eventos adversos (EA) causados durante a prestação de cuidados à saúde. A partir dessa exposição, governos e organizações mundiais motivaram-se a apoiar estratégias para a prevenção e a mitigação de falhas no cuidado à saúde, a fim de minimizar e/ou evitar os Erros Adversos (EA) (Brasil, 2017).

Evento adverso é definido pelo Ministério da Saúde como todo e qualquer incidente que resulte em dano ao paciente (Brasil, 2013). Em países desenvolvidos, constatou-se a ocorrência de EA em 4 a 16\% de pacientes hospitalizados. No Brasil os dados são alarmantes, como aponta pesquisa conduzida no estado do Rio de Janeiro (RJ), no qual foi observado que dos 1.103 pacientes adultos internados obteve-se incidência de EA em 7,6\% e a proporção de EA evitáveis de 66,7\%, sendo uma das maiores identificadas no mundo (Maia, Freitas, Gallo \& Araújo, 2018).

A alta prevalência de EA constatada, tanto no Brasil como em todo o mundo, aponta a segurança do paciente como uma importante questão de saúde pública e alerta para a necessidade de políticas para sua melhoria (Aranaz-Andrés et al., 2011). Diante desse engajamento mundial na busca pela identificação e erradicação dos riscos aos pacientes, em 2013 o Brasil institui o Programa Nacional de Segurança do Paciente (PNSP), que tem por objetivo geral contribuir para a qualificação do cuidado em todos os estabelecimentos de saúde do território nacional (Brasil, 2013). 
Ainda de acordo com a Portaria $\mathrm{n}^{\circ}$ 529, de $1^{\circ}$ de abril de 2013, fica instituído, no âmbito do Ministério da Saúde, o Comitê de Implementação do Programa Nacional de Segurança do Paciente (CIPNSP), instância colegiada, de caráter consultivo, com a finalidade de promover ações que visem à melhoria da segurança do cuidado em saúde através de processo de construção consensual entre os diversos atores que dele participam (Brasil, 2013).

Um dos objetivos do CIPNSP é a validação de protocolos, guias e manuais voltados à segurança do paciente e a OMS é o órgão que se responsabiliza por selecioná-los e validá-los, levando em consideração o baixo investimento necessário para a sua implantação e a relevância dos erros e eventos adversos decorrentes da ausência deles (Brasil, 2014).

Diante da motivação e necessidade de se desenvolver estudos sobre Segurança do Paciente (SP), as ações de formulações de protocolos, guias e manuais, foram refinadas a seis áreas prioritárias, denominadas Metas Internacionais para Segurança do paciente (Silva et al., 2017).

A sexta Meta Internacional para Segurança do Paciente faz referência a prevenção dos riscos de lesão por pressão (LPP), como eventos adversos de alta incidência, que estão intrinsecamente ligados a assistência direta ao paciente, sendo consideradas um grande desafio principalmente para a equipe de enfermagem, exigindo que esta seja qualificada para identificar os fatores de risco, planejar e implementar medidas eficazes para a prevenção e tratamento (Brasil, 2017).

É sempre importante valorizar o incentivo às práticas voltadas a SP que em todos os ambientes das instituições hospitalares, porém, em UTI, onde os EA são singularmente preocupantes, uma vez que o perfil de gravidade clínica resultam em maior risco ao paciente e, consequentemente, aumento significativo da mortalidade e da permanência hospitalar do cliente prejudicado pelo dano, torna-se pedra angular para desencadear novas formas de olhar e instigar delineamentos mais efetivos de cuidado (Ortega, D'Innocenzo, Silva \& Bohomol, 2017).

A incidência de LPP em clientes de UTI atinge de 23,1\% a 59,5\% de ocorrência. Com isso, tendo alto potencial danoso, a LPP requer completa atenção por parte da equipe de saúde assistência, enfatizando a atuação da enfermagem, uma vez que, a prevenção desse malefício envolve cuidados diretos e individualizados ao cliente hospitalizado. (Fernandes,Silva, Oliveira, Souza \& Nicola, 2016).

Portanto, o aparecimento da LPP em pacientes internados pode ser utilizado como um indicador de qualidade dos cuidados prestados pela equipe de enfermagem, cabendo a ela identificar os fatores de risco para o desenvolvimento da LPP nos clientes e planejar ações de caráter preventivo (Santos \& Lino, 2018).

Nesse ínterim, a assistência de enfermagem sem suporte teórico, conceitual, científico e padronizado desencadeia um exercício profissional imperito, negligente ou imprudente, podendo acarretar danos aos pacientes, problemas legais e éticos aos profissionais e descrédito da classe pela sociedade (Pimenta et al., 2015).

Portanto, estudos defendem que o uso de protocolos pode ser um aliado importante para a melhoria da qualificação dos profissionais mediante a tomada de decisão assistencial, pois proporciona a disseminação de conhecimento, a comunicação profissional e a coordenação do cuidado, favorecendo assim, a uma assistência prestada mais segura.

Sendo o protocolo a descrição de uma situação específica de assistência e/ou de um cuidado, que contém detalhes operacionais, orientações específicas sobre o que se faz, quem faz e como se faz, conduzindo e orientando os profissionais nas decisões de assistência para a prevenção, recuperação ou reabilitação da saúde, acredita-se que o uso dessa ferramenta tende a aprimorar a assistência, favorecer o uso de práticas cientificamente sustentadas, minimizar a multiplicidade das informações e condutas entre os membros da equipe de saúde, estabelecer limites de ação e cooperação entre os diversos profissionais, além de serem instrumentos construídos dentro dos princípios da prática baseada em evidências e oferecerem as melhores condutas de cuidado (Pimenta et al, 2015).

Nesse contexto, esta pesquisa objetivou averiguar o conhecimento de enfermeiros intensivistas acerca da prevenção e avaliação de risco relacionados a LPP e a importância da utilização de protocolos de cuidados de enfermagem. 


\section{Metodologia}

Trata-se de estudo exploratório-descritivo, com abordagem qualitativa realizado na UTI do Hospital Municipal de Natal (HMN), Rio Grande do Norte (RN) / Brasil. A instituição tem cunho estritamente público e possui sistema de atendimento de urgência com regulação prévia, composta por cerca de 80 leitos de enfermaria e 10 leitos de UTI.

Foram incluídos no estudo os enfermeiros especialistas assistenciais que atuam na UTI do HMN, juntamente com a enfermeira coordenadora do setor e gerente de enfermagem, além das enfermeiras responsáveis pela Comissão de Curativos e Segurança do Paciente, totalizando dez participantes. Não fizeram parte da pesquisa aqueles enfermeiros que estavam de férias ou afastados das atividades laborais por licença médica.

Os dados foram coletados entre março e dezembro de 2019. Para tal, foram aplicadas entrevistas semiestruturadas individuais, de forma remota através do Google Meet, gravadas através da mesma plataforma e realizadas em locais previamente combinados a fim de propiciar o máximo de conforto ao entrevistado e tempo necessário para a sua efetivação. Para assegurar a participação dos profissionais selecionados para a pesquisa, foram disponibilizados para serem lidos e assinados o Termo de Consentimento Livre e Esclarecido, bem como os termos de uso de voz e imagem, ambos enviados via e-mail, nos quais foram inseridos as assinaturas digitais dos entrevistados, além de serem esclarecidas as prováveis dúvidas em relação aos objetivos da pesquisa e seu desenvolvimento metodológico.

O instrumento aplicado nessa fase foi constituído por três partes a saber: a primeira parte buscou identificar o conhecimento dos enfermeiros acerca da utilização da Escala de Braden (EB). A segunda parte se deteve a investigação do nível de conhecimento e práticas dos profissionais acerca da prevenção e avaliação dos riscos de LPP nos pacientes internados na UTI e a terceira e última parte fez referência ao conhecimento dos profissionais sobre a importância e utilização de protocolos de cuidados de enfermagem.

Posteriormente, as respostas dos entrevistados foram transcritas na íntegra e analisadas através do Discurso do Sujeito Coletivo (DSC), conceituado como um discurso-síntese redigido na primeira pessoa do singular, composto pelas expressõeschaves $(\mathrm{ECH})$, que são trechos literais do discurso que revelam a essência do depoimento, as ECH devem ter a mesma ideia central (Lefévre \& Lefévre, 2003).

O diferencial da metodologia do DSC é que estão associados os conteúdos das opiniões de sentidos semelhantes presentes em diferentes depoimentos, de modo a formar com tais conteúdos um depoimento síntese, como se tratasse de uma coletividade falando na pessoa de um indivíduo (Lefévre \& Lefévre, 2014).

Por se tratar de uma pesquisa com seres humanos, a mesma foi norteada pela Resolução 466/12 (Brasil, 2013b), aprovada pelo Conselho Nacional de Saúde do Ministério da Saúde e no Código de Ética dos Profissionais de Enfermagem. Ademais, o estudo foi analisado e aprovado pelo Comitê de Ética em Pesquisa em Seres Humanos da Universidade Federal do Rio Grande do Norte (CEPSH/UFRN) através da Plataforma Brasil, parecer nº 3.755.918.

\section{Resultados}

A análise do material empírico foi baseada nas respostas de seis questionamentos realizados aos participantes do estudo, que resultaram em nove discursos coletivos importantes para melhor compreender o objeto de estudo proposto, sempre respeitando o rigor científico necessário para o desenvolvimento da metodologia escolhida. As respostas foram subdivididas em duas categorias, A e B, quando dois tipos de respostas diferentes foram obtidos. Com isso, os questionamentos 1, 2 e 3 apresentam DSC nas categorias A e B, enquanto os questionamentos 4, 5 e 6 apenas apresentam DSC na "Categoria A". 
Os resultados obtidos acerca dos conhecimentos dos enfermeiros sobre prevenção de LPP durante a sua formação acadêmica, deram origem a duas categorias distintas de DSC, a "Categoria A" demonstrou satisfação com os princípios adquiridos em suas formações e a "Categoria B" total insatisfação desses profissionais em relação a essa construção de conhecimento prévia, como observado abaixo:

Questão 1 - Durante sua formação, você teve contato com os conhecimentos sobre prevenção de LPP?

DSC 1 - Categoria A (Satisfatório na Graduação): Sim, eu tive contato sobre o assunto durante minha graduação, tanto na teoria quanto na prática com os estágios durante o curso, também foi abordado sobre as formas de prevenção e também sobre as formas de tratamento.

DSC 1 - Categoria B (Insatisfatório na Graduação): Sim, tive pouco contato com esse assunto, de forma muito simples, não dão tanta importância, muito mais teórico do que prático. Meu maior aprendizado não foi na graduação, e sim durante meus estágios em que eu tive bastante contato com esse tema e consegui aprender mais, só que algumas coisas mudaram bastante.

Quando questionados acerca da EB na facilitação do cuidado diário na UTI, também obtivemos duas categorias distintas de DSC, a "Categoria A" refere-se aos profissionais que concordam que a EB é facilitadora do cuidado, enquanto a "Categoria B" demonstrou que a EB nãos é facilitador do cuidado. Observamos as duas categorias nos discursos abaixo.

Questão 2 - Como o (a) Sr (a) acha que a EB pode facilitar o cuidado diário na UTI? O (a) Sr (a) poderia me explicar por quê?

DSC 1 - Categoria A (EB é facilitadora do cuidado): Eu acho que a escala facilita no sentido de mudar o olhar pro paciente, a escala de Braden foi desenvolvida para mensurar o quão aquele paciente é suscetível a apresentar LPP, é muito simples e didática, qualquer profissional de enfermagem pode avaliar e mensurar um cuidado a partir do uso dessa escala. Eu acredito que ela é de extrema importância para realmente ser utilizada no ambiente de terapia intensiva como cuidado diário para guiar o profissional, uma vez que, seu uso ajuda a predizer o risco da lesão do paciente, auxiliando a enfermagem a trabalhar de forma sistematizada e individualizada. A escala de Braden facilita que toda equipe possa usar a mesma forma de avaliação, auxiliando a programar nossas ações.

DSC 2 - Categoria B (EB não é facilitadora do cuidado): A escala de Braden, funciona para mim como um mero formalismo, os profissionais não verificam o que de informação aquela escala tá trazendo em relação ao risco de desenvolver lesão por pressão nos pacientes, no meio de tantas atribuições ela se torna apenas o preenchimento obrigatório de um papel. A escala de Braden poderia ser muito mais bem 
utilizada, porém, infelizmente eu vejo que o enfermeiro, não consegue colocar em prática a avaliação produzida, por isso a escala por si só não traz muitos resultados para a prevenção de LPP na UTI.

Em relação às dificuldades encontradas para implantar o DSC 3 "Categoria A" denota não encontrar dificuldade e a "Categoria B" refere-se encontrar dificuldade na implantação das práticas de avaliação de riscos e prevenção de LPP. As duas categorias do DSC estão expostas abaixo.

Questão 3 - O (a) Sr (a) encontra alguma dificuldade para implantar as práticas de avaliação de riscos e prevenção de LPP? Explique-me quais são elas?

DSC 3 - Categoria A (Não encontro dificuldades): Não vejo dificuldades em implantar as práticas, mas sim, sinto falta das ações dos enfermeiros para prevenção das lesões por pressão após a avaliação diária da pele do paciente.

DSC 3 - Categoria B (Encontro dificuldades): As dificuldades são inúmeras, mas as principais estão relacionadas a falta de materiais essenciais para a prevenção das lesões, como: coxins, lençóis e coberturas específicas para o tratamento das lesões, bem como, também nos submetemos a recursos humanos no limite do dimensionamento, o que dificulta uma boa assistência, há resistência da própria equipe de enfermagem em realizar as mudanças de decúbito.

De acordo com a opinião dos profissionais sobre a definição do protocolo de cuidados, foi obtido apenas uma categoria de resposta onde todos concordavam que o protocolo de cuidados contribui positivamente para a assistência.

Questão 4 - Para o(a) $\operatorname{Sr}(a)$ o que é um protocolo de cuidados? Comente um pouco sobre isso.

DSC 4 - Categoria A (O protocolo de cuidados contribui positivamente para a assistência): Protocolo de cuidados é um documento sistematizado que visa orientar, unificar e padronizar o cuidado, buscando minorar riscos e eventos adversos e proporcionando uma assistência mais assertiva. É criado com embasamento científico, por especialistas que buscaram conhecimento nas melhores literaturas, para que a enfermagem realize todos os cuidados de forma completa.

Assim como no DSC 4, o DSC 5 só obteve uma categoria de respostas. O questionamento referese em qual a opinião dos profissionais enfermeiros em como um protocolo de cuidados pode ajudar a prevenir LPP, as respostas foram unânimes concordando que o protocolos facilitaria a prática assistencial.

Questão 5 - Para o (a) Sr (a) Como um Protocolo de cuidados pode ajudar a prevenir as LPP em um ambiente de UTI?

DSC 5 - Categoria A (Sim): O Protocolo de cuidados facilitaria a prática assistencial, padronizando condutas, ajudando a equipe a trabalhar de forma única e falando a mesma língua, deixando a equipe 
com conhecimento homogêneo. Dessa forma, auxiliaria na prevenção da lesão por pressão pois direcionaria o olhar do profissional para o melhor acompanhamento do paciente, priorizando a continuidade da assistência. Esse protocolo traria mais força para a enfermagem, seria uma maior segurança, uma maior confiabilidade nas ações que seriam realizadas, pois são baseados nas melhores evidências científicas.

Para finalizar, o questionamento 6 trata da opinião dos profissionais sobre se concordo ou discorda com uma afirmação sobre se um protocolo de cuidados criado pela própria equipe de trabalho teria um diferencial, para isso, foi gerado apenas uma categoria já que todos os profissionais concordaram com a afirmação. Segue abaixo a abordagem desse resultado.

\section{Questão 6 - Agora, eu vou dizer uma frase e quero saber se o(a) $\operatorname{Sr}$ (a) concorda ou discorda e por quê. A frase é: Um protocolo de cuidados criado pela própria equipe de trabalho é um diferencial em relação a segurança do paciente. Por quê?}

DSC 6 - Categoria A (Concordo): Concordo, pois a equipe que trabalha diretamente naquele setor tem mais propriedade para elencar os cuidados possíveis frente a realidade vivida, os profissionais conhecem suas limitações e fragilidades e são capazes de contribuir para a criação desse instrumento, além de conhecerem as necessidades do setor e estarem envolvidos naquele contexto. A criação do protocolo pela equipe irá integrar o que precisa ser feito com o que é disponibilizado pela instituição. Vale ressaltar a importância de que essa equipe também tenha a perspicácia, a questão técnica, o conhecimento, faça buscas na literatura mais atual para fazer um protocolo realmente de qualidade e que possa conseguir atingir o seu propósito que é prevenir que esse paciente venha a desenvolver lesão por pressão.

\section{Discussão}

No DSC 1, os profissionais discorrem sobre o contato com os conhecimentos sobre prevenção de LPP durante sua formação acadêmica. Desta forma, estudos demonstram que o perfil de profissionais da saúde vem sofrendo modificações, voltados atualmente para uma formação que contemple conhecimento, habilidades e atitudes em vista por serem compreendidas como estratégias indispensáveis para um processo de formação elevado, garantindo posteriormente, um desempenho profissional diferenciado de forma qualificada e segura (França et al., 2019).

Assim, percebemos a importância de os profissionais em formação serem nutridos desde o princípio da jornada acadêmica com conhecimentos, competências e práticas baseadas em evidência, para que tenham subsídios e estejam aptos a implementação durante a prática profissional (Mazzo et al., 2018; França et al., 2019). Porém, pesquisas relatam (Sousa \& Faustino, 2019; Galvão, Serique, Santos \& Nogeira, 2017; Campoi et al., 2019) que enfermeiros apresentam dificuldade em realizar avaliações adequadas de pele e feridas e que este déficit está relacionado a falta de experiência e treinamento.

Portanto, apesar da formação acadêmica adequada ser fundamental, torna-se necessário a implementação de estratégias de educação continuada como o desenvolvimento de treinamentos e atualizações para que os profissionais 
enfermeiros exerçam cotidianamente com competência, qualidade e segurança (França et al., 2019; Mazzo et al., 2018; Sousa, 2019; Galvão et al., 2017; Campoi et al., 2019).

Quanto a opinião dos entrevistados sobre se a EB pode facilitar o cuidado diário na UTI podemos ver duas opiniões divergentes. A primeira, concorda que a EB é facilitadora do cuidado prestado, o que corrobora com estudos realizados em outras instituições hospitalares (Araújo \& Santos, 2016; Adamczyk et al., 2017; Sousa, França \& Jesus, 2016; Cardoso et al., 2019). Pesquisas apontam que a EB propicia aos profissionais uma melhor percepção dos fatores agressores ao paciente colaborando, por conseguinte, para a implementação efetiva de medidas preventivas as LPP além de auxiliar na identificação correta de problemas e no estabelecimento de prioridades (Araújo \& Santos, 2016).

Ademais, pesquisa recentes concordam que não existe um protocolo ou escala universalmente implementada e aceita como o melhor método para a realização de avaliações ao paciente em UTI, porém a unanimidade entre os especialistas é que não haja restrição e confiança cega apenas nas pontuações de instrumentos norteadores, mas sim que os profissionais da saúde tenham senso crítico para avaliar outros fatores influenciadores, de forma que a assistência prestada seja adaptado as individualidades do indivíduo e ao local do profissional enfermeiro (Adamczyk et al., 2017; Sousa, França \& Jesus, 2016).

Entretanto, uma segunda opinião como DSC foi elencada, a de que a EB não é facilitadora do cuidado. Com isso, percebemos em outros estudos que em algumas situações, os enfermeiros consideram a EB apenas como um documento a ser preenchido de forma obrigatória, burocrática e automatizada (Debon, Fortes, Rós, Scaratti, 2018). Desta forma, é necessário o pensamento da criação de uma cultura de segurança dentro dos estabelecimentos de saúde, implementando instrumentos validados, treinando e capacitando o profissional sobre a utilização e importância de escalas para avaliação de riscos, garantindo deste modo que escalas de avaliação sejam implementados como colaboradores ao cuidados, não substituindo a avaliação clínica do enfermeiro (Santos, 2017; Debon et al., 2018; Adamczyk et al., 2017; Sousa, França \& Jesus, 2016).

O DSC 3 refere-se às dificuldades para a implementação das práticas de avaliação de riscos e prevenção de LPP. Destacamos neste momento estudos que corroboram com as opiniões sobre a existência de dificuldades na implementação de avaliações, ressaltando obstáculos quanto a falta de recursos humanos tanto em quantitativo insuficiente, como também em escassez de adesão e disposição da equipe aos treinamentos e capacitações; e desprovimento nos insumos materiais para promoção do conforto e a segurança no posicionamento adequado (Araujo \& Santos 2016; Campoi et al., 2019).

Por obviedade, se faz necessário o fornecimento de condições apropriadas de trabalho, seja referente ao dimensionamento de profissionais quanto a qualificação dos mesmo e a disponibilidade de recursos físicos e materiais propícios a prestação de uma assistência qualificada evitando assim, ter-se uma equipe profissional desmotivada ocasionando uma assistência propícia a ocorrência de riscos e eventos adversos evitáveis (Campoi et al., 2019).

A abordagem referente a importância do protocolo de cuidados na prevenção de LPP foi elucidado nos DSC 4 e 5 com unanimidade entre os profissionais. Fortalecendo esse posicionamento, observa-se estudos que avaliaram as ações dos profissionais de enfermagem antes e após a implementação de protocolo de prevenção de LPP em UTI, concluindo que após a utilização do protocolo, constatou-se maior periodicidade das ações preventivas, confirmando a relevância no uso de ferramentas e protocolos como implementação de recomendações baseadas em evidência científicas (Vargas, 2019).

Todavia, o estudo de Mendonça (2018) revelou que a EB era preenchida de forma indiscriminada, evidenciado pela uniformização da prática dos profissionais quanto a ações de prevenção de LPP e a elaboração de prescrições de enfermagem iguais para os sujeitos independente das classificações de risco e avaliação clínica. Dessa maneira, a avaliação de pacientes internados em UTI deve ser fundamentada, criteriosa e detalhada abrangendo fatores de risco existentes e potenciais para LPP (Mendonça, Loureiro, Frota \& Souza, 2018).

Por fim, o DSC 6 versa sobre protocolos desenvolvidos pela própria equipe que presta assistência ao sujeito e se esse instrumento teria diferencial em relação a segurança do paciente. Logo, pesquisadores apontam que é possível a criação ou 
adaptação de protocolos através de pilares como diálogo e comprometimento conjunto entre profissionais e gestores das instituições. A criação e implementação de um protocolo pela equipe de enfermagem pressupõe a participação ativa dos profissionais que atuam na assistência ao sujeito, resultando em um protocolo mais condizente com as particularidades do serviço, resultando em apoio e estímulo frente às mudanças e melhorias no trabalho cotidiano das equipe de saúde, qualificando a assistência prestada (Figueiredo et al., 2018; Krauzer, Dall’Agnoll, Gelbcke, Lorenzini \& Ferraz, 2018).

\section{Conclusão}

Atendendo o objetivo proposto, o estudo possibilitou uma melhor compreensão das condições vivenciadas pelos enfermeiros que atuam em UTI apontando potencialidades, dificuldades e limitações na prevenção e tratamento as LPP.

Com isso, foi notório o potencial dos profissionais para o desenvolvimento de protocolos que se adequem a prática assistência em suas diversas particularidades porém medidas devem ser adotadas e direcionados para o incentivo à adesão ao uso de protocolos e sua importância no cuidado a saúde para que os mesmo não sejam vistos apenas como preenchimento obrigatório, com o intuito de gerar mudança na cultura dos profissionais, bem como adequar os recursos físicos e infraestrutura, garantindo assim melhor qualidade da assistência prestada. A preocupação com a LPP deve ser constante dentro dos serviços de saúde, com o objetivo de garantir a qualidade e segurança do cuidado, propiciando ambientes mais seguros para pacientes, familiares, acompanhantes e profissionais, buscando reduzir o número de IH e, consequentes, dos custos hospitalares gerados por esse dano.

Desta forma, este estudo demonstra a importância da assistência de enfermagem na prevenção de afecções evitáveis, como a Lesão por pressão e o indispensável uso da EB de forma rotineira. Vale ressaltar a relevância de novos estudos voltados a essa temática visando a disseminação do conhecimento e aprimoramento de um cuidado baseado em evidência.

\section{Referências}

Adamczyk, S. P., Castro, E. C. L. S., Freitas, T. M., Santos, W. B., Marques, F. R., \& Kutzke, J. L. (2017). Métodos utilizados pela enfermagem na identificação da lesão por pressão: uma revisão sistemática da literatura. Revista Gestão \& Saúde, 17, 1-9.

Agência Nacional de Vigilância Sanitária. (2017). Assistência Segura: Uma Reflexão Teórica Aplicada à Prática Agência Nacional de Vigilância Sanitária. Brasília (DF); 2017. http://www.saude.pi.gov.br/uploads/divisa_document/file/374/Caderno_1_-_Assist\%C3\%AAncia_Segura__Uma_Reflex\%C3\%A3o_Te\%C3\%B3rica_Aplicada_\%C3\%A0_Pr\%C3\%A1tica.pdf

Aranaz-Andrés, J. M., Aibar-Remón, C., Limón-Ramírez, R. A., Amarilla, A., Restrepo, F. R., Urroz, O., \& Gonseth-García, J. (2011). Prevalence of adverse events in the hospitals of five Latin American countries: results of the 'Iberoamerican study of adverse events' (IBEAS). BMJ Quality \& Safety, 20(12), 10431051 .

Araújo, A. A., \& dos Santos, A. G. (2016). Úlceras por pressão em pacientes internados em unidades de terapia intensiva: revisão integrativa da literatura. Ciência \& Saúde, 9(1), 38-48.

Campoi, A. L. M., Engel, R. H., Stacciarini, T. S. G., Cordeiro, A. L. P. D. C., Melo, A. F., \& Rezende, M. P. (2019). Permanent education for good practices in the prevention of pressure injury: almost-experiment. Revista brasileira de enfermagem, 72(6), 1646-1652.

Cardoso, D. D. S., Carvalho, F. M. O., Rocha, G. B. D., Mendes, J. R., Cardoso, S. D. B., \& Rocha, F. C. V. (2019). Conhecimento dos enfermeiros sobre classificação e prevenção de lesão por pressão. Rev. pesqui. cuid. fundam, 560-566.

Conselho Regional de Enfermagem. (2015). Guia para construção de protocolos assistenciais de enfermagem. São Paulo (SP): COREN-SP; 2015. https://portal.coren-sp.gov.br/sites/default/files/Protocolo-web.pdf.

Debon, R., Fortes, V. L. F., Rós, A. C. R., \& Scaratti, M. (2018). The nurses' viewpoint regarding the use of the braden scale with the elderly patient. Revista de Pesquisa: Cuidado é fundamental online, 10(3), 817-823.

Fernandes, L. M., da Silva, L., de Oliveira, J. L. C., de Souza, V. S., \& Nicola, A. L. (2016). Association between pressure injury prediction and biochemical markers. Revista da Rede de Enfermagem do Nordeste, 17(4), 490-497.

Figueiredo, T. W. B., Mercês, N. N. A. D., Lacerda, M. R., \& Hermann, A. P. (2018). Developing a nursing healthcare protocol: a case report. Revista Brasileira de Enfermagem, 71, 2837-2842.

França, A. P. F.M., Castro Rassy, M. E., Portilho, R. D. C. B., Montalvão Serrão, A. C. F., França, A. S., \& Silva Miranda, E. D. S. (2019). Conhecimento de enfermeiros sobre o manejo de lesões por pressão em unidade de terapia intensiva. Revista Eletrônica Acervo Saúde, 11(8), e576-e576. 
França, J. R. G., Saousa, B. V. N., \& Jesus, V. S. (2016). Cuidados de enfermagem na prevenção de lesões por pressão em unidades de terapia intensiva: uma revisão sistemática. Revista Brasileira de Saúde Ocupacional, 11(1), 16-31.

Galvão, N. S., Serique, M. A. B., Santos, V. L. C. D. G., \& Nogueira, P. C. (2017). Knowledge of the nursing team on pressure ulcer prevention. Revista brasileira de enfermagem, 70(2), 294-300.

Krauzer, I. M., Dall'Agnoll, C. M., Gelbcke, F. L., Lorenzini, E., \& Ferraz, L. (2018). The construction of assistance protocols in nursing work. REME Rev min enferm. [internet], 22, 1-9.

Lefèvre, F., \& Lefèvre, A. M. C. (2003). O discurso do sujeito coletivo: um novo enfoque em pesquisa qualitativa; desdobramentos. In $O$ discurso do sujeito coletivo: um novo enfoque em pesquisa qualitativa; desdobramentos (pp. 255-255).

Maia, C. S., Freitas, D. R. C. D., Gallo, L. G., \& Araújo, W. N. D. (2018). Notificações de eventos adversos relacionados com a assistência à saúde que levaram a óbitos no Brasil, 2014-2016. Epidemiologia e Serviços de Saúde, 27, e2017320.

Mazzo, A., Miranda, F. B. G., Meska, M. H. G., Bianchini, A., Bernardes, R. M., \& Pereira Junior, G. A. (2018). Teaching of pressure injury prevention and treatment using simulation. Escola Anna Nery, 22(1).

Mendonça, P. K., Loureiro, M. D. R., Frota, O. P., \& Souza, A. S. D. (2018). Prevenção de Lesão por Pressão: ações prescritas por enfermeiros de centros de terapia intensiva. Texto \& Contexto-Enfermagem, 27(4).

Ministério da Saúde. (2014). Documento de referência para o Programa Nacional de Segurança do Paciente. Brasília (DF); 2014. http://bvsms.saude.gov.br/bvs/publicacoes/documento_referencia_programa_nacional_seguranca.pdf

Ministério da Saúde. (2013). Portaria n 529 de $1^{\text {o }}$ de abril de 2013. Institui o Programa Nacional de Segurança do Paciente (PNSP). Brasília (DF); 2013a, http://bvsms.saude.gov.br/bvs/saudelegis/gm/2013/prt0529_01_04_2013.html

Ministério da Saúde. (2013). Protocolo para prevenção de úlcera por pressão. Brasília (DF); 2013b. https://www20.anvisa.gov.br/segurancadopaciente/index.php/publicacoes/item/ulcera-por-pressao

Ortega, D. B., D’Innocenzo, M., Silva, L. M. G. D., \& Bohomol, E. (2017). Análise de eventos adversos em pacientes internados em unidade de terapia intensiva. Acta Paulista de Enfermagem, 30(2), 168-173.

Paim, L., Trentini, M., Madureira, V. S. F., \& Stamm, M. (2008). Pesquisa convergente-assistencial e sua aplicação em cenários da enfermagem. Cogitare Enfermagem, 13(3).

Santos, L. R. C. L., \& Lino, A. (2018). Riscos de lesão por pressão: aplicação da Escala de Braden em terapia intensiva. ESTIMA. Braz. J. Enterostomal Ther, 16(818), 1-7.

Silva C. B., Silva D. G., Carvalho L. L., Goulart C. D. L., Silva A. L. G., \& Angri D. (2017). Ocorrência de eventos adversos em Unidade de Terapia Intensiva Neopediátrica: qualidade assistencial de enfermagem. Rev Epidemiol Control Infect, 1;7(4).

Sousa, B. V. N., França, J. R. G., \& Jesus, V. S. (2016). Cuidados De Enfermagem Na Prevenção De Úlceras Por Pressão Em Unidades De Terapia Intensiva: Uma Revisão Sistemática. Revista brasileira de saúde funcional, 1(2), 16-16.

Sousa, R. C., \& Faustino, A. M. (2019). Nurses’ understanding about the pressure injury prevention and care/Conhecimento de enfermeiros sobre prevenção e cuidados de lesão por pressão. Revista de Pesquisa: Cuidado é Fundamental Online, 11(4), 992-997.

Vargas, R. G., \& Santos, L. P. (2019). Prevenção de lesão por pressão em UTI-aplicabilidade da Escala de Braden. Revista Pró-UniverSUS, 10(1), 162-165. 